\title{
Modified constraint-induced movement therapy for clients with chronic stroke: interrupted time series (ITS) design
}

\author{
JuHyung Park, MSc, OT ${ }^{1,2)}$, NaYun Lee, MSc, OT ${ }^{1,2)}$, YongHo Cho, PhD, $\mathrm{PT}^{3)}$, \\ YeONGAE YANG, PhD, $\left.\mathrm{OT}^{4}\right)^{*}$ \\ 1) Department of Occupational Therapy, Kyungbuk College, Republic of Korea \\ 2) Department of Rehabilitation Science, Graduate School, Inje University, Republic of Korea \\ 3) Department of Physical Therapy, Daegu Haany University, Republic of Korea \\ 4) Department of Occupational Therapy, College of Biomedical Science and Engineering, Inje \\ University: 197 Inje-ro, Gimhae-si, Gyyeongsangnam-do 219-705, Republic of Korea
}

\begin{abstract}
Purpose] The purpose of this study was to investigate the impact that modified constraint-induced movement therapy has on upper extremity function and the daily life of chronic stroke patients. [Subjects and Methods] Modified constraint-induced movement therapy was conduct for 2 stroke patients with hemiplegia. It was performed 5 days a week for 2 weeks, and the participants performed their daily living activities wearing mittens for 6 hours a day, including the 2 hours of the therapy program. The assessment was conducted 5 times in 3 weeks before and after intervention. The upper extremity function was measured using the box and block test and a dynamometer, and performance daily of living activities was assessed using the modified Barthel index. The results were analyzed using a scatterplot and linear regression. [Results] All the upper extremity functions of the participants all improved after the modified constraint-induced movement therapy. Performance of daily living activities by participant 1 showed no change, but the results of participant 2 had improved after the intervention. [Conclusion] Through the results of this research, it was identified that modified constraint-induced movement therapy is effective at improving the upper extremity functions and the performance of daily living activities of chronic stroke patients.

Key words: Constraint-induced movement therapy, Interrupted time series design, Stroke
\end{abstract}

(This article was submitted Sep. 2, 2014, and was accepted Nov. 7, 2014)

\section{INTRODUCTION}

Constraint-induced movement therapy (CIMT) is an intervention for stroke patients that has been suggested to promote the movement of the upper extremities on the paralyzed side. It is an intensive rehabilitation treatment method that promotes the use of the unused affected side by restricting the less-affected side, which is the side that is primarily used in daily life ${ }^{1)}$. Although many previous studies have demonstrated the effectiveness of CIMT at recovering the upper extremity function of stroke patients, there are many issues regarding its practical clinical use, since it restricts the movement of the less-affected upper extremity by the wearing of assistive devices such as splints or gloves for $90 \%$ of the time spent performing daily living activities over a 2 to 3 week span, and the patients suffer from the excess time taken

*Corresponding author. YeongAe Yang (E-mail: Share5184@, naver.com)

(C2015 The Society of Physical Therapy Science. Published by IPEC Inc. This is an open-access article distributed under the terms of the Creative Commons Attribution Non-Commercial No Derivatives (by-ncnd) License $<$ http://creativecommons.org/licenses/by-nc-nd/3.0/>. to perform their daily living activities, as well as psychological anxiety and pressure arising from intensive treatment of the paralyzed side for 6 to 7 hours a day ${ }^{2-4)}$.

To minimize the limitations of CIMT, many forms of modified CIMT (m-CIMT) have been designed. The most typical form consists of restricting the movement of the less-affected side of the upper extremities for 5 hours a day, 5 times a week for 10 weeks, in combination with an intensive training session for the affected side of the upper extremity 3 times a week ${ }^{2}$. Another design consists of restricting the movement of the less-affected side of the upper extremity for 6 hours a day, 5 times a week for 2 to 3 weeks and conducting intensive training for 2 hours ${ }^{5,6}$. Many previous studies have reported that chronic stroke patients exhibit improvement in the functional capabilities of the upper extremities, performance of daily living activities, and quality of life when m-CIMT was conducted for them ${ }^{7,8)}$. However, there were many studies of chronic stroke patients in which the number of participants was too low, and, in terms of experimental design, the majority of studies only consisted of simple comparisons of results before and after the intervention. It is difficult to treat this as an analysis that considers the many variables that can affect general therapy environments and influence the results of an experiment. It 
Table 1. General characteristics of the subjects

\begin{tabular}{lccccccc}
\hline & Age & Gender & Diagnosis & $\begin{array}{c}\text { Affected } \\
\text { side }\end{array}$ & $\begin{array}{c}\text { Dominant } \\
\text { hand }\end{array}$ & $\begin{array}{c}\text { Month from } \\
\text { stroke onset }\end{array}$ & MMSE-k \\
\hline Participant 1 & 68 & female & ischemic & Lt. & Rt. & 60 & 26 \\
Participant 2 & 47 & male & ischemic & Rt. & Rt. & 38 & 27 \\
\hline
\end{tabular}

is also difficult to differentiate the recovery of participants that would have occurred under normal conditions from the recovery that occurred due to an intervention ${ }^{9}$.

The interrupted time series (ITS) design is an experimental design that is appropriate for experiments that have a low number of participants, and it investigates the functional and performance abilities of the participants over a set period of time before and after an intervention, rather than measuring them at a certain point in time before and after an intervention, in order to compare the trend of recovery under normal conditions with recovery after an intervention through the comparison of the trend lines of the recovery of each patient based on the results obtained before and after intervention ${ }^{9)}$. Hence, this study used the ITS design to understand the therapeutic effects of m-CIMT when it was conducted for chronic stroke patients.

\section{SUBJECTS AND METHODS}

The subjects were 2 patients who were hospitalized in one hospital in Korea after being diagnosed with hemiplegia due to stroke. The general characteristics of the subjects are noted in Table 1.

Participant 1 was a 47-year-old male who had right hemiplegia due to middle cerebral artery infarction that had occurred 48 months earlier. Participant 2 was a 68 -year-old female who had left hemiplegia due to middle cerebral artery infarction that had occurred 60 months earlier. Both participants had used their right hand as their dominant hand before stroke, and they were participating in 30 minutes each of conservative physical therapy and occupational therapy 5 times a week. The subjects and their guardians signed an informed consent form after receiving information about the purpose and method of the study. The selection criteria for the participants were as follows: no m-CIMT or related treatments in the past two years, the ability to stand without falling when the less-affected side was restricted, a score of 25 points or more on Mini-Mental Status Examination-Korea (MMSE-K) indicating absence of cognitive damage, no restriction of passive joint range of motion, and some amount of active joint range in motion exercises. This study was conducted after receiving approval of its safety, procedures, and ethics from the Research Ethics Committee of Daegu Haany University.

This study used an interrupted time series (ITS) design to determine whether CIMT had an effect greater than that of natural recovery on the impairment of the hemiplegic arm and hand of elderly persons after stroke. ITS designs are characterized by the collection of multiple observations over time that are "interrupted" by an intervention or treatment ${ }^{10)}$. An ITS design enables the researcher to quantify a stable baseline level of function or a trend before treatment and then project that trend into the future. The effect of the treatment is determined as the difference between the actual and projected outcomes ${ }^{11)}$. This study followed the Effective Practice and Organization of Care Cochrane definition of an ITS design, which requires that there be at least three time points before and after the intervention, that the intervention occurs at a clearly defined point in time, and that the study uses objective performance or outcome measures ${ }^{9)}$.

The assessment of the participants was conducted 5 times over 3 weeks before and after the m-CIMT intervention. The assessment tools chosen were those that are most frequently used in real clinical environments and easily accessible. These included the modified Barthel index (MBI) for assessing the daily living activity capabilities of the participants, a hand-held dynamometer to measure their grip strength and assess their upper extremity functional levels, and the box and block test (BBT). As one of the tools that assesses the daily living activity capabilities of stroke patients, MBI is widely used for its convenience, high accuracy, consistency, sensitivity, and ease of statistical processing compared to other assessment tools ${ }^{12)}$. BBT is a standardized assessment tool that assesses the agility of the upper extremities. It is simple to assess since it measures the number of wooden blocks moved in one minute. The test-retest reliability of this tool was reported as 0.93 for the left hand and 0.97 for the right, and the reliability between testers was reported as 0.99 for the left hand and 1.00 for the right ${ }^{13)}$. The handheld dynamometer was used to measure grip strength, which was measured with the participants sitting on a chair, with their elbow joint flexed at $90^{\circ}$ and the forearm in the neutral position. The participants were asked to squeeze the handle tightly on the tester's verbal order and were then given some rest time to recover from fatigue. The measurement was repeated three times each and the mean value was calculated $^{14)}$.

The m-CIMT program designed for the participants was conducted 5 times a week for 2 weeks, and it comprised 6 hours in which the participants wore a resting splint in their normal life to restrict the movement of the less-affected side. The 6 hours included the 2 hours of participation in the therapy program. The activities used in the therapy program included those that participants came across frequently in their daily life, such as turning over book pages, writing words, moving cones, opening a toothpaste cap and squeezing the toothpaste out, cutting paper with scissors, putting on and taking off socks, etc. The patients were asked to perform various activities with objects of various sizes and shapes.

Data analysis included descriptive analysis of mean differences and trend line analysis to test whether the modified CIMT had a larger positive effect on impairment than natural recovery. For each participant, individual preintervention data points were used to determine the mean 
pre-intervention score of each measure. The same procedure was followed to obtain the mean post-intervention scores. The pre-intervention scores were also used to create trend lines for each participant. Trend lines were calculated using the Excel 2010 scatterplot function, and linear regression was used to predict the post-intervention result if no change was expected. Ninety-five percent confidence intervals (CIs) were calculated for each data point. The predicted trend lines were then compared with the actual post-intervention results in terms of slope and in terms of the overlap of $95 \%$ CIs for each data point. A significant change was considered to have occurred if the CIs did not overlap ${ }^{15)}$.

\section{RESULTS}

The before, after, and mean difference scores of both measures are presented in Table 2. Positive changes were observed in most of the items for all participants, and only the MBI scores of participant 2 showed no difference between pre- and post-intervention.

For both participants, the post-intervention trend lines for the score of the BBT and grip strength was above the predicted trend lines. For both of the participants, there was no overlap in CIs between the predicted and post-intervention BBT score and grip strength trend lines, indicating improvements associated with treatment rather than natural recovery.

The MBI scores before and after the intervention of participant 1 were identical, resulting in overlapping trend lines. For participant 2, the post-intervention trend lines for the score of the MBI was above the predicted trend lines. For participant 2, there was no overlap in CIs between the predicted and post-intervention MBI trend lines, indicating improvements associated with treatment rather than natural recovery.

\section{DISCUSSION}

The primary purpose of this study was to evaluate whether modified CIMT had a greater effect on the upper extremity function and ADL than natural recovery of two chronic phase stroke patients. The results of this study confirmed the positive effect of m-CIMT for all items except the MBI of participant 1.

For five days a week over a 2 week span, the participants of this study had restriction of the less-affected side for only 4 hours during normal daily activities, excluding the 2 hours they spent during the therapy program. Using a 16 hours as the patients' waking activity time, 6 hours, corresponding to $37.5 \%$ of the waking hours of the day is considerably less than the times used for CIMT in previous studies. Constraint of the less affected side was applied 5 days a week for 2 weeks, and included 2 hours of therapy programs. Despite this, positive effects on the functional capabilities of the affected side were observed for most of the evaluation measures used in this study ${ }^{8}$. One possible explanation for why we were able to see a strong effect is that the high-intensity program of massed practice may be more important than motor restriction of the less affected arm, as suggested by Liepert et al. and Taub et $\mathrm{al}^{16,17)}$.

The results of the study reveal there were positive im-
Table 2. Mean pre- and post-intervention scores and mean difference

\begin{tabular}{lccc}
\hline Measure & $\begin{array}{c}\text { Pre- } \\
\text { intervention } \\
\text { mean }\end{array}$ & $\begin{array}{c}\text { Post- } \\
\text { intervention } \\
\text { mean }\end{array}$ & $\begin{array}{c}\text { Mean } \\
\text { difference }\end{array}$ \\
\hline BBT & & & \\
Participant 1 & 13.0 & 19.0 & 6.0 \\
Participant 2 & 12.6 & 15.2 & 2.6 \\
Grasp power & & & \\
Participant 1 & 9.1 & 10.8 & 1.7 \\
Participant 2 & 3.3 & 6.6 & 3.3 \\
MBI & & & \\
Participant 1 & 81.0 & 81.0 & - \\
Participant 2 & 72.0 & 76.0 & 4.0 \\
\hline
\end{tabular}

provements in the upper extremity function of both participants, which is in agreement with the results of Dromerick et al. who reported that the upper extremity function of the affected side improved when m-CIMT was conducted for chronic stroke patients ${ }^{18)}$. However, there was no change in the daily living activity capabilities of participant 1 . We think this was because the participant 1 was older than participant 2, and because the affected side of participant 1 was her dominant side before the disease; the affected side of participant 2 was his dominant side before the disease. The present results are in agreement with those of Paik Young-rim and Kim Soo-kyoung who reported that, due to the nature of m-CIMT, which restricts the less-affected side and promotes the use of the affected side, recovery of the neurologic pattern of the dominant hand (primarily used in functional tasks before the disease) would be quicker since modification of learned non-use in the dominant hand is easier than in the non-dominant hand ${ }^{7}$.

The clinical significance of this research is that it has verified a valid research method from the experimental design aspect furthering the work of previous studies that conducted m-CIMT for chronic stroke patients. However, considering the small test group, for further research and for the generalization of study results, it will be necessary to perform a statistically meaningful experiments with a minimum number of 30 experimental subjects.

\section{REFERENCES}

1) Taub E, Miller NE, Novack TA, et al.: Technique to improve chronic motor deficit after stroke. Arch Phys Med Rehabil, 1993, 74: 347-354. [Medline]

2) Page SJ, Levine P, Sisto $S$, et al.: Stroke patients' and therapists' opinions of constraint-induced movement therapy. Clin Rehabil, 2002, 16: 55-60. [Medline] [CrossRef]

3) Pierce SR, Gallagher KG, Schaumburg SW, et al.: Home forced use in an outpatient rehabilitation program for adults with hemiplegia: a pilot study. Neurorehabil Neural Repair, 2003, 17: 214-219. [Medline] [CrossRef]

4) Sterr A, Elbert T, Berthold I, et al.: Longer versus shorter daily constraintinduced movement therapy of chronic hemiparesis: an exploratory study. Arch Phys Med Rehabil, 2002, 83: 1374-1377. [Medline] [CrossRef]

5) Cope SM, Forst HC, Bibis D, et al.: Modified constraint-induced movement therapy for a 12-month-old child with hemiplegia: a case report. Am J Occup Ther, 2008, 62: 430-437. [Medline] [CrossRef]

6) Yu JH, Kang HK, Jung JH: Effects of modified constraint-induced movement therapy on hand dexterity, grip strength and activities of daily living 
of children with cerebral palsy: a randomized control trial. J Phys Ther Sci, 2012, 24: 1029-1031. [CrossRef]

7) Paik YR, Kim SK: A task-oriented approach consisting of modified constraint-induced movement therapy (m-CIMT) and bimanual activity effects on upper extremity function and activities of daily living (ADL) in stroke patients. J Korean Soc Occup Ther, 2010, 18: 79-94.

8) Choi YI, Jung MY, Chung BI, et al.: The effect of constraint-induced movement therapy on the affected upper extremity function and activities of daily living for stroke patients. J Korean Soc Occup Ther, 2009, 17: 77-90.

9) Ramsay CR, Matowe L, Grilli R, et al.: Interrupted time series designs in health technology assessment: lessons from two systematic reviews of behavior change strategies. Int J Technol Assess Health Care, 2003, 19: 613-623. [Medline] [CrossRef]

10) Cook TD, Campbell DT: Quasi-experimentation: Design and Analysis Issues for Field Settings. Chicago: Rand McNally, 1979.

11) Johnston MV, Ottenbacher KJ, Reichardt CS: Strong quasi-experimental designs for research on the effectiveness of rehabilitation. Am J Phys Med Rehabil, 1995, 74: 383-392. [Medline] [CrossRef]
12) Smith A: Beware of the Barthel. Physiotherapy, 1993, 79: 843-844. [CrossRef]

13) Cromwell FS: Occupational Therapist Manual for Basic Skill Assessment: Primary Pre-vocational Evaluation. Altaden: Fair Oaks Printing Co., 1976.

14) Mathiowetz V, Weber K, Volland G, et al.: Reliability and validity of grip and pinch strength evaluations. J Hand Surg Am, 1984, 9: 222-226.

15) Borg WR, Gall MD: Educational research: An introduction, 5th ed. New York: Longman, 1989

16) Yong MS, Hwangbo K: Skilled reach training influences brain recovery following intracerebral hemorrhage in rats. J Phys Ther Sci, 2014, 26: 405-407. [Medline] [CrossRef]

17) Taub E, Crago JE, Uswatte G: Constraint induced movement therapy: a new approach to treatment in physical rehabilitation. Rehabil Psychol, 1998, 43: 152-170. [CrossRef]

18) Dromerick AW, Edwards DF, Hahn M: Does the application of constraintinduced movement therapy during acute rehabilitation reduce arm impairment after ischemic stroke? Stroke, 2000, 31: 2984-2988. [Medline] [CrossRef] 